This article is licensed under the Creative Commons Attribution-NonCommercialNoDerivatives 4.0 International License (CC BY-NC-ND) (http://www.karger.com/Services/ OpenAccessLicense). Usage and distribution for commercial purposes as well as any distribution of modified material requires written permission.

\title{
Invasive Pulmonary Adenocarcinoma with Lepidic Growth Pattern in a Pregnant Patient
}

\author{
Omar Jiménez-Zarazúa ${ }^{a, b} \quad$ Lourdes N. Vélez-Ramírez \\ José C. Padilla-López ${ }^{c}$ Juana R. García-Ramírez ${ }^{\text {b, d }}$ \\ Pedro L. González-Carillo ${ }^{e}$ Jaime D. Mondragón ${ }^{f, g}$ \\ aHospital General León, Department of Internal Medicine, León, Mexico; bUniversidad de \\ Guanajuato, Department of Medicine and Nutrition, León, Mexico; 'Hospital General León, \\ Department of Radiology, León, Mexico; ${ }^{\mathrm{d} H o s p i t a l}$ General León, Department of

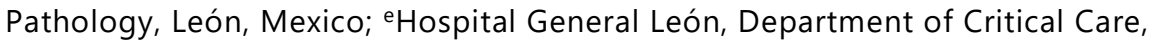 \\ León, Mexico; fUniversity of Groningen, University Medical Center Groningen, Department \\ of Neurology, Groningen, The Netherlands; GUniversity of Groningen, University Medical \\ Center Groningen, Alzheimer Research Center, Groningen, The Netherlands
}

\section{Keywords}

Acute respiratory failure - Adenocarcinoma - Alveolar infiltrate - Lepidic growth - Lung cancer . Pregnancy

\section{Abstract}

Among the differential diagnoses that should be considered in acute respiratory failure (ARF) are infectious processes, autoimmune diseases, interstitial pulmonary fibrosis, and pulmonary neoplasia. Timely diagnosis of lung neoplasia is complicated in the early stages. An opportune diagnosis, as well as the specific treatment, decrease mortality. ARF occurs 1 in 500 pregnancies 


\section{Case Reports in Oncology}

Case Rep Oncol 2018;11:822-834

DOI: $10.1159 / 000495460$

(c) 2018 The Author(s). Published by S. Karger AG, Basel www.karger.com/cro

Jiménez-Zarazúa et al.: Invasive Pulmonary Adenocarcinoma with Lepidic Growth Pattern in a Pregnant Patient

and is most common during the postpartum period. Among the specific etiologies that cause ARF during pregnancy that must be considered are: (1) preeclampsia; (2) embolism of amniotic fluid; (3) peripartum cardiomyopathy; and (4) trophoblastic embolism. The case of a 36-yearold patient with a 33-week pregnancy and ARF is presented. The patient presented dyspnea while exerting moderate effort that progressed to orthopnea and type 1 respiratory insufficiency. Imaging studies showed bilateral alveolar infiltrates and predominantly right areas of consolidation. Blood cultures, a galactomannan assay and IgG antibodies against mycoplasma pneumoniae, were reported as negative. Autoimmune etiology was ruled out through an immunoassay. A percutaneous pulmonary biopsy was performed and an invasive pulmonary adenocarcinoma with lepidic growth pattern (i.e. lepidic pulmonary adenocarcinoma, LPA) result was reported. This etiology is rare and very difficult to recognize in acute respiratory failure cases. After infectious, autoimmune and interstitial lung fibrosis have been excluded the clinician must suspect of lung cancer in a patient with acute respiratory failure and chest imaging compatible with the presence of ground-glass nodular opacities, a solitary nodule or mass with bronchogram, and lung consolidation. In the presence of acute respiratory failure, the suspicion of pulmonary neoplasia in an adult of reproductive age must be timely. Failure to recognize this etiology can lead to fatal results.

(C) 2018 The Author(s)

Published by S. Karger AG, Basel

\section{Introduction}

Acute respiratory failure (ARF) is a rare clinical entity during pregnancy, occurring in 1 in 500 pregnancies [1]. In ARF, the inadequate gas exchange may be due to pulmonary and extrapulmonary conditions. ARF can produce type 1 (i.e. hypoxemic) or type 2 (i.e. hypercapnic) respiratory failure. Specific causes of ARF in pregnancy include etiologies such as: (1) abrupt placenta; (2) embolism of amniotic fluid; (3) sepsis (e.g. chorioamnionitis); (4) preeclampsia (e.g., secondary pulmonary edema); and (5) trophoblastic pulmonary embolism [1]. Among the factors that may increase the risk of ARF are viral pneumonitis, non-obstetric sepsis, asthma, air embolism, thromboembolic disease, vascular disease, and pulmonary hypertension [1]. Other non-specific medical conditions that are associated with ARI are trauma, pancreatitis, and neuromuscular disorders [1]. The clinical picture of an ARF varies according to the etiology. At the onset, ARF can commence as an increase in respiratory rate associated with accessory respiratory muscle use (i.e. scalene and sternocleidomastoid muscles) or as tachycardia and is often associated with signs related with to adrenergic release (e.g. diaphoresis) [2]. Treatment for ARF should be directed toward attending the underlying disorder, ensuring proper airway management, assessing respiratory pattern, and adequate circulatory perfusion [2]. The medical relevance of this case report pertains to the opportune screening for lung cancer in cases of acute respiratory failure where infectious, autoimmune and interstitial pulmonary fibrosis have been excluded. 


\section{Case Reports in Oncology}

\begin{tabular}{l|l}
\hline Case Rep Oncol 2018;11:822-834 \\
\hline DOI: 10.1159/000495460 & $\begin{array}{l}\text { (c) } 2018 \text { The Author(s). Published by S. Karger AG, Basel } \\
\text { www.karger.com/cro }\end{array}$
\end{tabular}

Jiménez-Zarazúa et al.: Invasive Pulmonary Adenocarcinoma with Lepidic Growth Pattern in a Pregnant Patient

\section{Case Presentation}

A 36-year-old female with a 33-week pregnancy arrived at the Emergency Department complaining of a dry cough and shortness of breath during the last four days. A couple of hours before seeking medical help, the patient presented dyspnea when exerting moderate effort (i.e. ascending approximately eight steps) and heart palpitations that subsided with rest. The respiratory distress had a torpid evolution, as the dyspnea progressed to orthopnea before seeking medical attention. Additionally, the patient presented generalized malaise (i.e. myalgia and arthralgia). The patient's family history included a mother with type 2 diabetes and arterial hypertension; other relevant aspects of family history were questioned and denied. Among the patient's personal history, she reported tobacco consumption for more than 5 years of approximately five cigarettes per day (i.e. 1.25 pack-year smoking history), suspending its consumption during the pregnancy. The patient denied use of controlled substances, allergies, past blood transfusions, traveling to regions with endemic diseases within the last three months, tattoos and body piercings. The patient had no history of lung disease, asthma during childhood, and chronic or degenerative diseases. The patient reported four pregnancies, one delivery, and two cesarean surgeries, without any complications in regards to her past obstetric history. At the moment of her Emergency Department admission, the patient reported a 33-week pregnancy with normal evolution.

Upon initial physical exploration, we found a recumbent patient with freely chosen body position, Glasgow coma score of 15, without focal neurologic deficits nor meningeal sings, aware of his environment, with reference to place, time, and people. The patient was diaphoretic, with skin and mucosae dehydrated $+/+++$. The head and neck exploration had no alterations. Upon inspection, the respiratory apparatus with oral ventilation, along with tachypnea associated with thoracic and abdominal dissociation. The thorax had decreased expansion without vibrations or fremitus during palpation. No asymmetries or abnormal findings in tone intensity, pitch, duration, and quality through direct percussion. Upon auscultation, disseminated bilateral crepitant crackles and bilateral decreased inspiratory breath sounds were found at the bases. Precordial auscultation revealed tachycardia and heart sounds of good intensity without extra heart sounds. The abdominal exploration presented a globous abdomen secondary to a gestating uterus with a live single product (i.e. a fetal heart rate of $158 \mathrm{bpm}$ ), without visceromegaly nor abnormalities upon light and deep palpation. The extremities had a filiform pulse augmented in frequency and decreased in amplitude without trophic changes. Upon admission, the patient had the following vital signs: BP of 130/90 mm Hg; HR of 100 bpm; $\mathrm{RR}$ of $21 \mathrm{rpm}$; $\mathrm{SO}_{2}$ with noninvasive ventilation at a rate of $10 \mathrm{~L} / \mathrm{min}$ of $74 \%$; temperature of $36^{\circ} \mathrm{C}$; body-weight of $70 \mathrm{~kg}$; height of $165 \mathrm{~cm}$; and BMI of 25.7 .

The patient developed type one respiratory insufficiency (i.e. vital signs: BP: $130 / 85 \mathrm{~mm}$ $\mathrm{Hg}$, HR: $120 \mathrm{bpm}, \mathrm{RR}: 36 \mathrm{rpm}$, Temp: $36.7^{\circ} \mathrm{C}$; arterial blood gas test: $\mathrm{pH}=7.4, \mathrm{PaO}_{2}=43 \mathrm{~mm}$ $\mathrm{Hg}, \mathrm{PaCO}_{2}=24 \mathrm{~mm} \mathrm{Hg}, \mathrm{HCO}_{3}{ }^{-}=16.9 \mathrm{mEq} / \mathrm{L}, \mathrm{O}_{2}$ content $=78 \%$, base excess $=-7.0 \mathrm{mmol} / \mathrm{L}$, lactate $=0.9 \mathrm{mmol} / \mathrm{L}$ ), while being under observation at the Emergency Department; consequently, requiring invasive artificial airway with an endotracheal tube. The patient presented thoracic and abdominal dissociation, intercostal retractions and diaphoresis. The patient was surgically intervened in a cesarean emergency procedure. A live newborn male of $1940 \mathrm{gr}$ was obtained, without malformations; additionally, a $400 \mathrm{cc}$ hemorrhage was reported during the 
surgical procedure. The patient was admitted to the Intensive Care Unit for further tests and treatment.

Laboratory results at admission are presented in Table 1 . On a chest x-ray, the posterioranterior projection shows bilateral opacities with predominance in the lower lobes (Fig. 1A). Bilateral alveolar infiltrates and areas of consolidation with a right predominance at the bases were evident in a computed tomography (CT) of the thorax (Fig. 2A-2D). Due to the clinical picture and imaging findings, an infectious process was suspected and was further explored. Antimicrobial treatment was initiated with one gram of intravenous (IV) ceftriaxone every 12 $\mathrm{h}$ and clarithromycin $500 \mathrm{mg}$ orally (PO) every $12 \mathrm{~h}$ for 7 days.

\section{Clinical Evolution}

Upon the patient's admission to the Intensive Care Unit, consecutive blood cultures of each extremity, a culture of bronchial secretions, IgG antibodies against Mycoplasma pneumonie and a galactomannan assay to exclude aspergillosis were performed; all being reported negative. Serum procalcitonin levels of $0.02 \mathrm{ng} / \mathrm{mL}$ were reported. The urinalysis reported abundant bacteria, erythrocytes 118 per field, as well as leukocytes of 163 per high power field (Table 2). In search of an autoimmune etiology, immunoassays were requested and reported as negative (Table 2).

Forty-eight hours after admission to the Intensive Care Unit the patient was extubated, presenting adequate respiratory mechanics. Due to the patient's clinical improvement and in absence of respiratory failure (i.e. arterial blood gas test: $\mathrm{pH}=7.46, \mathrm{PaO}_{2}=74.9 \mathrm{~mm} \mathrm{Hg}, \mathrm{PaCO}_{2}$ $=39 \mathrm{~mm} \mathrm{Hg}, \mathrm{HCO}_{3^{-}}=28 \mathrm{mEq} / \mathrm{L}, \mathrm{O}_{2}$ content $=95.6 \%$, base excess $=6.0 \mathrm{mmol} / \mathrm{L}$, lactate $=0.5$ $\mathrm{mmol} / \mathrm{L}$ ), but still dependent on supplemental oxygen, the patient was admitted to the Internal Medicine Department. An echocardiogram was performed reporting a left ventricular ejection fraction of $66 \%$ and normal systolic function; however, mild diastolic dysfunction, yet excluding a decompensated heart failure as a cause of the ARF. A global pericardial effusion with a posterior predominance of approximately 300 cubic centimeters was detected, without evidence of intracavitary thrombi, nor evidence of valvular pathology (Fig. 1C, D).

A CT-guided percutaneous lung biopsy was performed during the second week of hospitalization (Fig. 3A, B). The histopathological report reported moderately differentiated malignant neoplasia compatible with invasive pulmonary adenocarcinoma with lepidic growth pattern (Fig. 3C. D). Immunohistochemistry studies were performed (Table 2) integrating a pulmonary neoplasia diagnosis. As a complication of the invasive procedure, the patient presented a pneumothorax greater than $25 \%$ in the right hemithorax (Fig. 1B). A tube thoracostomy was performed, placing an endopleural tube on the on the right mid-axillary line to correct for the presence of pleural effusion. Pleural effusion was examined and the report described the presence of liquid compatible to exudate according to the Light criteria for pleural effusions.

The patient was evaluated by the Oncology Department, classifying the patient as completely disabled (i.e. ECOG 4) and making the determination that chemotherapy administration was contraindicated due to an unresolved sepsis process. Sepsis of unknown origin was integrated as follow-up urinalyses, urine culture and blood cultures were negative and no pulmonary septic process could be confirmed. Additionally, after her condition and prognosis 


\section{Case Reports in Oncology}

Case Rep Oncol 2018;11:822-834

DOI: $10.1159 / 000495460$

(c) 2018 The Author(s). Published by S. Karger AG, Basel www.karger.com/cro

Jiménez-Zarazúa et al.: Invasive Pulmonary Adenocarcinoma with Lepidic Growth Pattern in a Pregnant Patient

were explained to the patient, she did not accept any further medical assistance or interventions. After assessment by the Oncology Department, a palliative care protocol was initiated with the administration of morphine for dyspnea and pain control. The following two weeks the patient had a torpid clinical evolution (i.e. continued orthopnea, a temperature of $39^{\circ} \mathrm{C}$, and leukocytosis; Table 2). After a total of 30 days of hospitalization, the patient presented extreme bradycardia, progressing to asystole and death. The family of the patient did not wish for a necropsy to be performed.

\section{Discussion}

Performing the diagnosis of a neoplasm during pregnancy is a challenge for the clinician. Physiological adaptations associated with pregnancy may mask the symptoms suggestive of cancer [3]. This case report presents a pregnant patient with acute respiratory failure secondary to an invasive pulmonary adenocarcinoma with lepidic growth pattern. In this case report, through clinical exploration, laboratory and imaging studies, an infectious process, an autoimmune disease, and an interstitial lung fibrosis were excluded; therefore, after a CT-guided percutaneous lung biopsy, an invasive pulmonary adenocarcinoma with lepidic growth pattern diagnosis was confirmed through histopathological examination. The main objective of this case report is to contribute to the existing medical literature a case of ARF with an etiology difficult to diagnose, which is the case of pulmonary neoplasia in a pregnant patient.

Neoplasms associated with pregnancy are reported to occur in 1 out of every 1,000 pregnancies and more recently an incidence of 122.9 cancer cases for every 100,000 pregnancies [4]. Breast cancer, thyroid cancer, and lymphomas are cancers most commonly associated with pregnant women [5]. Lung cancer is divided into two types: (1) non-small cell lung cancer (NSCLC), which represents approximately 85\% of all lung cancers; and (2) small cell lung cancer, which represents the remaining 15\% [6]. Although progress regarding early detection of non-small cell lung cancer has been made, it is usually diagnosed at an advanced stage; thus, resulting in poor prognosis [6]. Weight loss, coughing, dyspnea, weakness, and chest pain are among the clinical manifestations of lung cancer [7]. The most frequent NSCLC is invasive lung adenocarcinoma, with five subtypes: (1) lepidic; (2) acinar; (3) solid; (4) papillary; and (5) micropapillary [8]. LPA is the most common form of adenocarcinoma proliferation, possessing a unique histological pattern termed lepidic growth. Lepidic growth is characterized by type II pneumocyte proliferation along the alveolar structure [9]. LPA affects primarily women, with the most frequent symptoms being: (1) weight loss (35.9-46\%); (2) coughing (4554.7\%); (3) dyspnea (37-45.3\%); (4) weakness (34\%); (5) chest pain (27\%) and (6) hemoptysis (17.4-27\%) [7]. According to a tumor resection study, most lung cancers present clinical symptoms in an advanced stage and surgical interventions are considered the 'gold' standard for the treatment of early NSCLC and some late NSCLC cases [9].

The patient presented in this case report only presented dyspnea, which progressed to orthopnea within a matter of days; developing type 1 respiratory insufficiency while being observed in the Emergency Department. Due to the acute onset of the respiratory failure, neoplasia was not the first etiology considered in this case's diagnostic algorithm. After assessment by the Obstetrics Department and given that the patient was intubated in order to provide adequate ventilation, the interruption of the pregnancy was decided by performing a 


\section{Case Reports in Oncology}

Case Rep Oncol 2018;11:822-834

DOI: $10.1159 / 000495460$

(c) 2018 The Author(s). Published by S. Karger AG, Basel www.karger.com/cro

Jiménez-Zarazúa et al.: Invasive Pulmonary Adenocarcinoma with Lepidic Growth Pattern in a Pregnant Patient

cesarean-type surgical intervention. The offspring's well-being was immediately procured by ending the pregnancy and no fetal suffering being reported. Adequate fetal oxygenation requires a $\mathrm{PaO}_{2}$ approximately of $70 \mathrm{~mm} \mathrm{Hg}$, which corresponds to a saturation of maternal $\mathrm{O}_{2}$ of around 95\% [10]. The effect of maternal hypercapnia on the fetus is not well defined. Limited research data on neonates reports that a $\mathrm{PaCO}_{2}$ of $44-55 \mathrm{~mm} \mathrm{Hg}$ can be well tolerated by the newborns [10].

Due to the gradual progression of the clinical picture, an infectious process was initially considered to be the cause of the patient's ARF. Our first diagnostic inquiry was substantiated on the clinical (i.e. orthopnea, myalgia, and arthralgia) and radiological findings (i.e. alveolar infiltrates and pulmonary consolidation) compatible with a pneumonic process. To exclude an infectious process, blood cultures of each extremity, a culture of bronchial secretions, IgG antibodies against Mycoplasma pneumonie and a galactomannan assay to exclude aspergillosis were performed and were reported negative. To investigate the cause of the pericardial effusion of approximately $300 \mathrm{cc}$ a transthoracic echocardiogram was performed to exclude a heart failure diagnosis. Only a mild diastolic failure was reported, which did not justify the patient's clinical picture.

A chest radiograph is often the first imaging modality to suggest the diagnosis of lung neoplasia and recent lung cancer screening guidelines recommend low-dose CT screening in certain cases [11]. However, none of the recent evidence-based recommendations for CT screening applied to the case presented; furthermore, highlighting the complexity of this case. Lung adenocarcinoma has three radiological patterns, as a solitary nodule (i.e. 60-90\%), as a dense consolidation (i.e. up to $20 \%$ ), and as multiple small sub-centimeter irregularly marginal nodules (i.e. rare) [12]. LPA is associated with a solitary nodule, which also is characterized by a ground-glass opacification pattern, mainly observed at the periphery of the lesion [12]. The case presented here had a dense condensation pattern resembling a pneumonic process and was also associated with a ground-glass opacification pattern. The pathological stage is the most important prognostic factor in patients with this pulmonary adenocarcinoma. The overall five year survival by pathological stage is $90,85,80,73,65,56,41,24$, and $12 \%$ for the IA1, IA2, IA3, IB, IIA, IIB, IIIA, IIIB, and IIIC according to the average overall survival in the International Association for the Study of Lung Cancer global database of patients receiving a diagnosis between 1999 and 2010. The case presented in this article is classified as a stage IIIA given its bilateral pulmonary disease through CT imaging and tissue biopsy [13].

\section{Limitations and Further Considerations}

One of the great limitations for an opportune diagnosis of pulmonary adenocarcinoma is the diagnostic accuracy of CT for this pathology. Aherne and colleagues (2018), report a series of cases that included 63 patients with pulmonary neoplasia with a lepidic component, where the radiological findings by CT were correlated with histopathological findings [14]. The authors reported an association between the size of the CT lesion and the histopathological diagnosis; specifically, a sensitivity of 0.719 (95\% CI: $0.533-0.863$ ) and a specificity of 0.704 (95\% CI: 0.498-0.862), when using the cut-off value for a lesion of $7.5 \mathrm{~mm}$ [14].

Pneumonia is among the first differential diagnosis for a radiological lung consolidation syndrome by CT. This was the initial diagnostic suspicion in the clinical case presented. Lymphoproliferative disorders (e.g. primary lymphoma of mucosa-associated lymphoid tissue) should also be excluded. These disorders usually present as a single nodule or consolidation 
opacity only in $33 \%$ of cases or as multiple nodules or consolidation of multiple areas in $43 \%$ of the cases [15]. Secondary lymphoma also has a similar presentation by CT; however, it is characterized by the presence of nodules and tumors with or without air bronchogram [15]. Among the lesions that may present ground-glass opacities are: focal interstitial fibrosis; aspergillosis; eosinophilic pneumonia; bronchiolitis obliterans with organizing pneumonia; thoracic endometriosis; focal traumatic lung injury; pulmonary Cryptococcus; inflammatory diseases, and focal hemorrhage. In this patient, serological detection of different antibodies was performed to exclude an immunological process before suspecting in a neoplasm.

Although lung tissue biopsy is an invasive diagnostic technique, it is a justified diagnostic measure in ARF cases where an infectious and autoimmune diagnosis has been excluded. Lung tissue biopsy can assist in the differential diagnosis between a neoplastic process and an interstitial pulmonary disease. Due to lack of access to bronchoscopy, a CT-guided biopsy was performed. A percutaneous image-guided biopsy is a safe and effective method to obtain a diagnosis of focal lesions. A percutaneous image-guided biopsy is a preferred technique for the diagnosis of new or growing lung nodules or when a bronchoscopy reports negative results for a persistent opacity of the airspace or hilar tumor.

\section{Conclusion}

In the presence of acute respiratory failure, the suspicion of pulmonary neoplasia in an adult of reproductive age must be timely. Lung cancer should be suspected in a patient with $\mathrm{ARF}$ and CT imaging compatible with the presence of ground-glass nodular opacities, a solitary nodule or mass with bronchogram, and lung consolidation. As part of the diagnostic algorithm of a patient of reproductive age, an infectious etiology, autoimmune etiologies (e.g. vasculitis and collagen disease), neoplastic processes (e.g. primary lymphoma, primary lymphoma of mucosa-associated lymphoid tissue and LPA), and interstitial pulmonary fibrosis should be excluded. The torpid evolution, in this case, is attributed to the concomitant sepsis process that the patient presented, which did not allow for treatment targeted for the lung cancer. The patient presented in this case had a locally advanced stage lung cancer corresponding to IIIA according to The Eighth Edition Lung Cancer Stage Classification; furthermore, at this stage, tumor resection is recommended. Among the therapeutic options for IIIA stage lung cancer are radiotherapy and chemotherapy session followed by adjuvant therapy with a secondary anti-neoplastic agent or if the epidermal growth factor receptor mutation is present Gefitinib, Erlotinib, Afatinib, Osimertinib, and Necitumumab can be used [16].

\section{Availability of Data and Materials}

The clinical data supporting the conclusions of this article is included in the article. 


\section{Acknowledgements}

This study was supported by CONACyT (Consejo Nacional de Ciencia y Tecnología) Grant \#440591. This research did not receive any specific grant from funding agencies in the commercial sector. We would like to commend the work of the medical staff (i.e. specialists, medical residents, and nursing staff) of the Internal Medicine Department at Hospital General León, radiology resident Felipe de Jesús Gaona and Oracio Gónzalez from the Cardiology Department at Hospital General León.

\section{Statement of Ethics}

Approval from the ethical committee was not required due to the nature of this case report. Abiding by the Declaration of Helsinki, patient anonymity was guaranteed. Upon hospital admission, the patient signed an informed consent permitting the use of her clinical file information for didactic and research purposes.

\section{Disclosure Statement}

The authors declare that there are no conflicts of interest relevant to this work.

\section{Author Contributions}

Study concept and design: OJZ, JDM. Analysis and interpretation of data: OJZ, LNVR, JCPL, PLGC, JRGR, and JDM. Drafting of the manuscript: OJZ and JDM. Critical revision of the manuscript for important intellectual content: All authors. All authors read and approved the final manuscript.

\section{References}

1 Lapinsky SE. Management of Acute Respiratory Failure in Pregnancy. Semin Respir Crit Care Med. 2017 Apr;38(2):201-7.

2 Creagh-Brown B. Respiratory failure. Medicine (Baltimore). 2016;44(6):342-5.

3 Davison J, Narain S, McEwan A. Cancer in pregnancy. Obstetrics, Gynaecol Reprod Med. 2017;27(8):251-5.

4 Parazzini F, Franchi M, Tavani A, Negri E, Peccatori FA. Frequency of Pregnancy Related Cancer: A Population Based Linkage Study in Lombardy, Italy. Int J Gynecol Cancer. 2017 Mar;27(3):613-9.

5 Herbst RS, Heymach JV, Lippman SM. Lung cancer. N Engl J Med. 2008 Sep;359(13):1367-80.

6 Kocher F, Hilbe W, Seeber A, Pircher A, Schmid T, Greil R, et al. Longitudinal analysis of 2293 NSCLC patients: a comprehensive study from the TYROL registry. Lung Cancer. 2015 Feb;87(2):193-200.

7 Travis WD, Brambilla E, Nicholson AG, Yatabe Y, Austin JH, Beasley MB, et al.; WHO Panel. The 2015 World Health Organization Classification of Lung Tumors: Impact of Genetic, Clinical and Radiologic Advances Since the 2004 Classification. J Thorac Oncol. 2015 Sep;10(9):1243-60.

8 Duruisseaux M, Antoine M, Rabbe N, Rodenas A, Mc Leer-Florin A, Lacave R, et al. Lepidic predominant adenocarcinoma and invasive mucinous adenocarcinoma of the lung exhibit specific mucin expression in relation with oncogenic drivers. Lung Cancer. 2017 Jul;109:92-100. 


\section{Case Reports in Oncology}

\begin{tabular}{l|l}
\hline Case Rep Oncol 2018;11:822-834 \\
\hline DOI: 10.1159/000495460 & $\begin{array}{l}\text { (c) 2018 The Author(s). Published by S. Karger AG, Basel } \\
\text { www.karger.com/cro }\end{array}$
\end{tabular}

Jiménez-Zarazúa et al.: Invasive Pulmonary Adenocarcinoma with Lepidic Growth Pattern in a Pregnant Patient

9 Weaver H, Coonar AS. Lung cancer: diagnosis, staging and treatment. Surgery. 2017;35(5):247-54.

10 Cole DE, Taylor TL, McCullough DM, Shoff CT, Derdak S. Acute respiratory distress syndrome in pregnancy. Crit Care Med. 2005 Oct;33(10 Suppl):S269-78.

11 Mazzone PJ, Silvestri GA, Patel S, Kanne JP, Kinsinger LS, Wiener RS, et al. Screening for Lung Cancer: CHEST Guideline and Expert Panel Report. Chest. 2018 Apr;153(4):954-85.

12 Ginsberg MS, Grewal RK, Heelan RT. Lung cancer. Radiol Clin North Am. 2007 Jan;45(1):21-43.

13 Detterbeck FC, Boffa DJ, Kim AW, Tanoue LT. The Eighth Edition Lung Cancer Stage Classification. Chest. 2017 Jan;151(1):193-203.

14 Aherne EA, Plodkowski AJ, Montecalvo J, Hayan S, Zheng J, Capanu M, et al. What CT characteristics of lepidic predominant pattern lung adenocarcinomas correlate with invasiveness on pathology? Lung Cancer. 2018 Apr;118:83-9.

15 Hirsch FR, Scagliotti GV, Mulshine JL, Kwon R, Curran WJ Jr, Wu YL, et al. Lung cancer: current therapies and new targeted treatments. Lancet. 2017 Jan;389(10066):299-311.

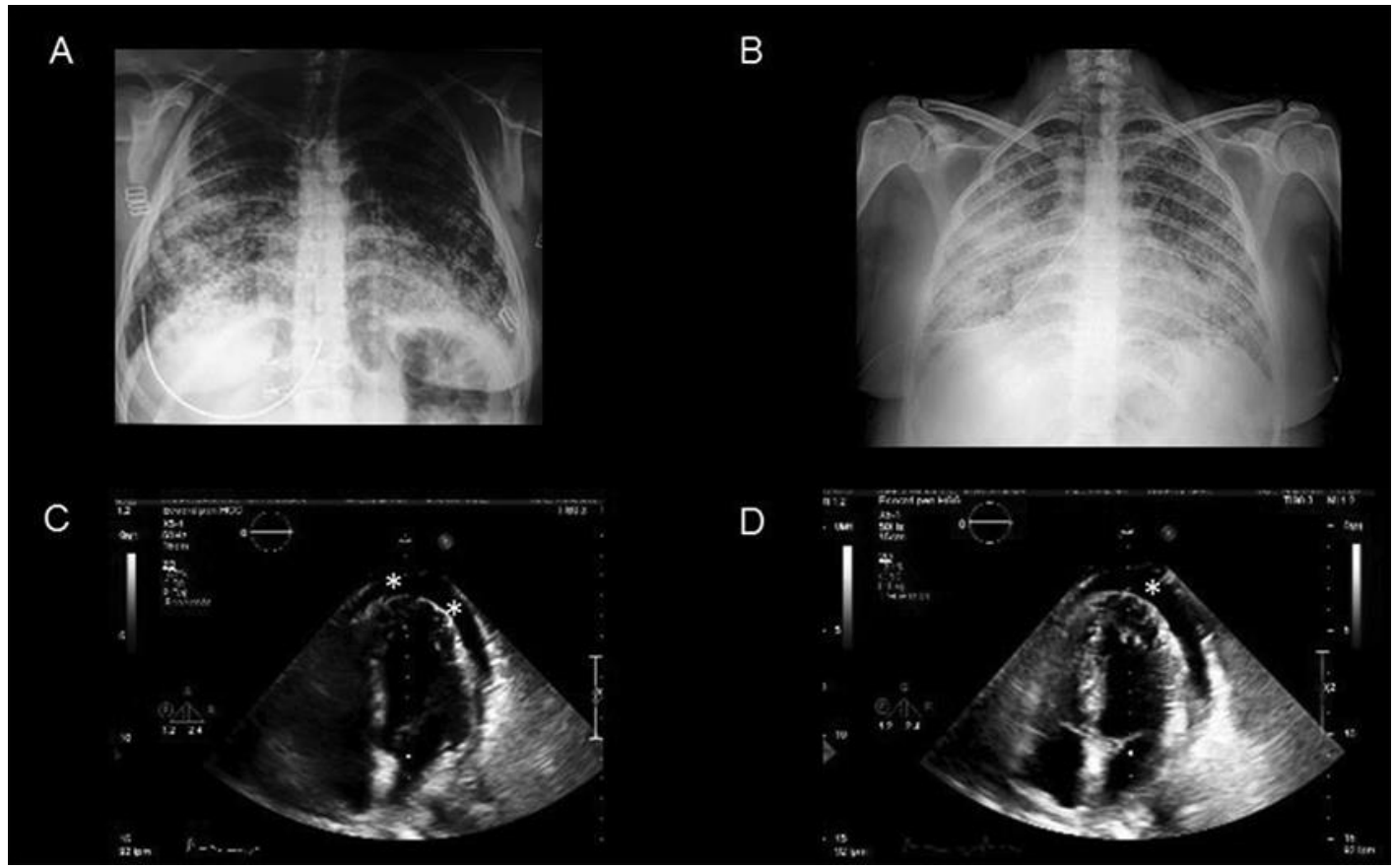

Fig. 1. Chest X-ray. Posterior-anterior projection. A) Bilateral opacities with predominance at the bases at admission. B) Bilateral alveolar infiltrates at the bases and areas of consolidation, with right predominance and bilateral progressive diffusion, compatible with ground-glass opacification images. The endopleural tube on the right side. Echocardiogram. Two-dimensional apical projection. C) Four chamber image showing a normal interventricular septum, without intracavitary thrombi or evidence of valvular pathology. D) Global pericardic effusion predominately posteriorly of approximately 300 cubic centimeters (marked by *). 


\section{Case Reports in Oncology}

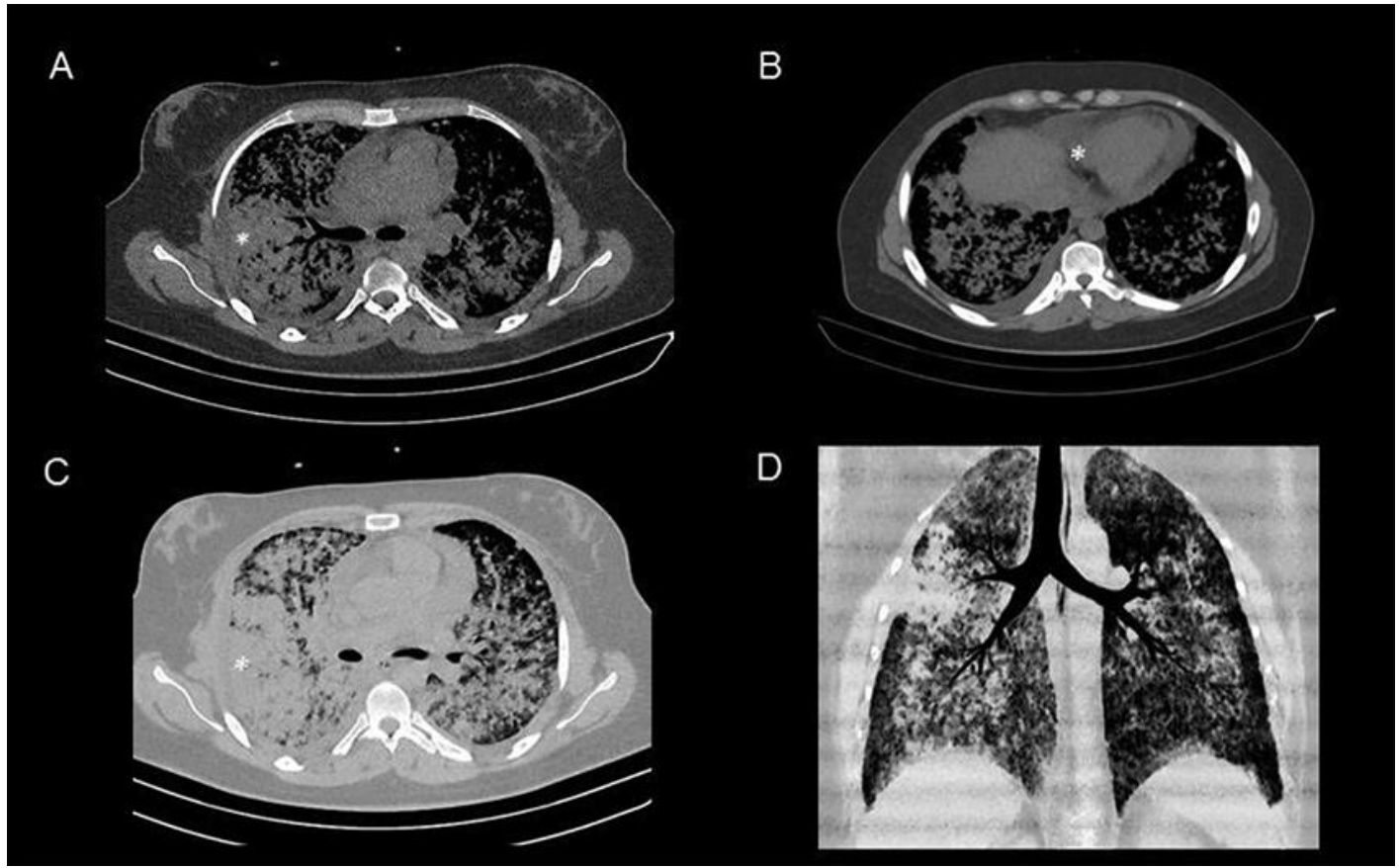

Fig. 2. Computerized tomography (CT) of the thorax. Thoracic CT scan without contrast. A) Bilateral alveolar infiltrates with consolidation area (marked by ${ }^{*}$ ) in the right lung region. B) Bilateral alveolar infiltrates and increased pericardic space due to pericardic effusion (marked by ${ }^{*}$ ). C) Consolidation area in right hemithorax area (marked by ${ }^{*}$ ). D) Consolidation area observed at the right superior lobule with multiple images compatible with ground-glass opacities and air bronchogram. 


\section{Case Reports in Oncology}

A

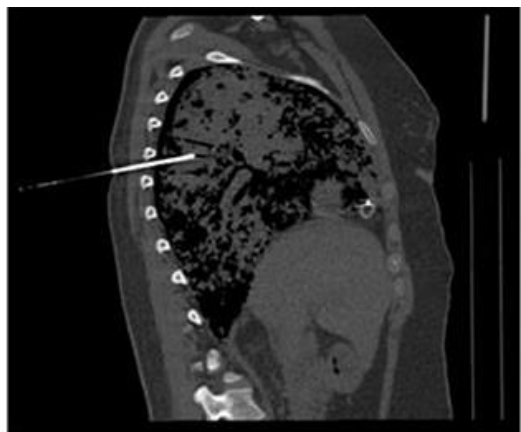

C

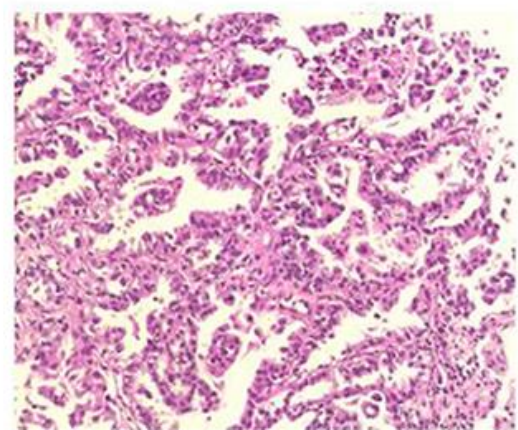

B

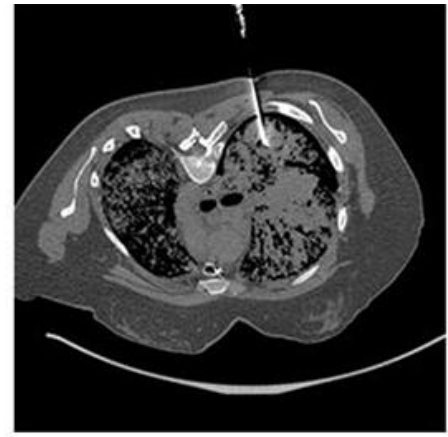

D

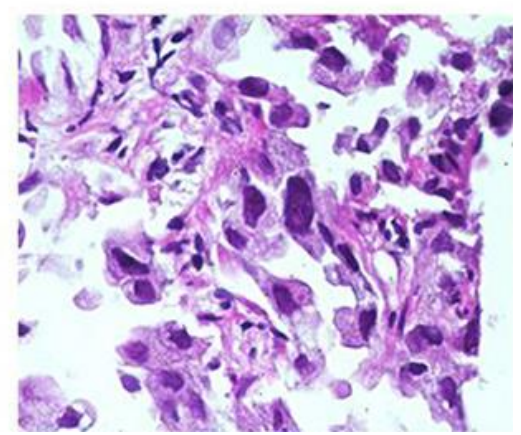

Fig. 3. Lung biopsy guided by CT. A) Biopsy needle at the fifth intercostal space in the dorsal aspect of the thorax. B) Biopsy needle at a mediastinal window. Histopathology. Lung. C) 10x, hematoxylin and eosin staining. Malignant neoplasm at the alveolar wall, partially respecting the alveolar lumen emitting projections resembling butterfly wings. D) 40x, hematoxylin and eosin staining. Alveolar wall coated with neoplastic cells that show marked pleomorphism, enlarged nuclei, and nuclear hyperchromatism. 
Table 1. Laboratory test results upon admission to the Emergency Department

Full blood count

Hemoglobin at admission

Hematocrit

Erythrocyte count

Platelet count

Mean corpuscular volume

DOI: $10.1159 / 000495460$

(c) 2018 The Author(s). Published by S. Karger AG, Basel www.karger.com/cro

Jiménez-Zarazúa et al.: Invasive Pulmonary Adenocarcinoma with Lepidic Growth Pattern in a Pregnant Patient

Mean corpuscular hemoglobin concentration

Leukocyte count

Lymphocytes

$14.3 \mathrm{~g} / \mathrm{dL}$

$43.1 \%$

$5.43 \times 10^{6} \mu \mathrm{L}$

$403 \mu \mathrm{L}$

$79.4 \mathrm{fL}$

$26.2 \mathrm{~g} / \mathrm{dL}$

Neutrophils

$11,000 \mu \mathrm{L}$

$10.4 \%$

$83.7 \%$

Monocytes

$5.4 \%$

$0.3 \%$

$0.2 \%$

Basophils

Blood chemistry

Glucose

Creatinine

$75.9 \mathrm{mg} / \mathrm{dL}$

$0.7 \mathrm{mg} / \mathrm{dL}$

$19 \mathrm{mg} / \mathrm{dL}$

Urea nitrogen

$8.8 \mathrm{mg} / \mathrm{dL}$

Blood urea nitrogen

$5.4 \mathrm{mg} / \mathrm{dL}$

Uric acid

Cholesterol

$150 \mathrm{mg} / \mathrm{dL}$

Triglycerides

$170 \mathrm{mg} / \mathrm{dL}$

Liver Function Enzymes

Aspartate transaminase

$33.1 \mathrm{U} / \mathrm{L}$

Alanine transaminase

$20.1 \mathrm{U} / \mathrm{L}$

Lactate dehydrogenase

$295.7 \mathrm{U} / \mathrm{L}$

Albumin

Alkaline phosphatase

Gamma-glutamyl transpeptidase

$3.1 \mathrm{~g} / \mathrm{dL}$

$163.8 \mathrm{U} / \mathrm{L}$

20

Blood coagulation

Prothrombine time

14.7 Sec

Partial thromboplastin time

$36.4 \mathrm{Sec}$

International normalized ratio

1.1

Electrolytes

Sodium

$137.8 \mathrm{mEq} / \mathrm{L}$

Potassium

$4.49 \mathrm{mEq} / \mathrm{L}$

Chlorine

$106.5 \mathrm{mEq} / \mathrm{L}$

Calcium

$8.3 \mathrm{mg} / \mathrm{dL}$

Phosphorus

$4.45 \mathrm{mg} / \mathrm{dL}$

Magnesium

$1.73 \mathrm{mg} / \mathrm{dL}$ 
Table 2. Supplementary laboratory test results

\section{Follow-up}

Full Blood Count at 4 weeks

Platelet count

Leukocyte count

Neutrophils

Lymphocytes

Monocytes

Eosinophils

Basophils

Procalcinotin

Antibodies

Cytoplasmic antineutrophil cytoplasmatic antibodies (cANCA)

Perinuclear antineutrophil cytoplasmatic antibodies (pANCA)

Anti-double-stranded deoxyribonucleic acid

Anti. SSB-LA

Anti -SSA-RO

Anti-SM

Anti-cardiolipin IgG

Anti-cardiolipin IgM antibody

Cyclic citrullinated peptide antibody

Complement C3

Complement C4

Viral panel

Hepatitis B virus

Hepatitis $C$ virus

Human immunodeficiency virus

Urinalysis at $72 \mathrm{~h}$

Appearance

$\mathrm{pH}$

Specific gravity

Proteins

Ketones, glucose, and nitrites

Leukocytes

Erythrocytes

Bacteria

\author{
$339,000 / \mu \mathrm{L}$ \\ $20,000 / \mu \mathrm{L}$ \\ $86.7 \%$ \\ $9.8 \%$ \\ $3.3 \%$ \\ $0 \%$ \\ $0.2 \%$ \\ $10 \mathrm{ng} / \mathrm{mL}$
}

0.1

0.2

$0.9 \mathrm{U} / \mathrm{mL}$

Negative

Negative

Negative

$2 \mathrm{U} / \mathrm{mL}$

$7.7 \mathrm{U} / \mathrm{mL}$

$1.5 \mathrm{U} / \mathrm{mL}$

$1.5 \mathrm{gr} / \mathrm{L}$

$0.5 \mathrm{gr} / \mathrm{L}$

Negative

Negative

Negative

Crystalline

6

1.020

Negative

Negative

163 per high power field 118 per high power field Abundant 